\title{
FUNCTIONAL OUTCOMES OF ACL RECONSTRUCTION USING HAMSTRING AUTO GRAFT.
}

1. MBBS, FCPS

Assistant Professor

Department of Orthopedic

Faisalabad Medical University \&

DHQ Hospital, Faisalabad.

2. MBBS M.Phil

Department of Physiology

Aziz Fatima Medical and

Dental College, Faisalabad.

3. MBBS M.Phil

Continental Medical College.

4. MBBS, MCPS

Department of Obs \& Gynae

Social Security Hospital.

5. MBBS M.Phil

Department of Physiology Aziz Fatima Medical and

Dental College, Faisalabad.

Correspondence Address:

Dr. Zeeshan Ali khan

P-238 Street No.7 Muslim Town 1

Sargodha Road, Faisalabad.

zeeshanalikhan68@gmail.com

Article received on:

02/05/2019

Accepted for publication:

05/07/2019

\begin{abstract}
Zeeshan Ali Khan ${ }^{1}$, Benash Altaf ${ }^{2}$, Samia Javed ${ }^{3}$, Sadaf Altaf ${ }^{4}$, Shireen Jawed ${ }^{5}$
\end{abstract}
ABSTRACT... Objectives: The knee joint is made of two cruciate ligaments. One is anterior cruciate ligament (ACL) which is weaker when it comes to comparison with the other cruciate ligament known as posterior cruciate ligament (PCL). $A C L$ tears are most common and frequently neglected. ACL tear is affecting $70 \%$ of the population and this high incidence reflects the significance of the problem. Reason of restoration of a torn ACL is to provide knee stability, knee motion in a safe wide range and to prevent osetoarthritis $\mathrm{OA}$. The gold standard for ACL auto-graft reconstruction is bone patella-tendon bone (BTB) which is still questioned by many researchers as this technique followed subjects suffered from knee pain. So aim is to use and to access outcomes of hamstring auto-graft for reconstruction ACL by using Tegner's score. Study Design: Cross sectional study. Setting: Private based hospital, Faisalabad. Period: January 2017 to January 2019. Material and Methods: It was comprised of in comprised of 97 subjects recruited on the base of positive Tegners score. Demographic data including age was presented as mean and standard deviation. Data was stratified for the variables i.e. age, gender, duration of disease and pre-operative Tegner activity rating scale to address the effect modifiers. Post-stratification Chi-square test is applied to check the significance with P-value less than 0.05 as significant. Regression was applied to check the effect of age on Tegner's scor. Results: This study comprised of 97 subjects ( 81 male, 16 females $83.5 .5 \%$ and $16.5 \%$ respectively from private setup based hospital with mean age of $31 \pm 11.1$. Our study showed $96.9 \%$ subjects with improved Tegner's scale after ACL reconstruction following hamstring autograph technique. Regression was also applied to check the effect of age on Tegner's score which was found to be significant ( $p$ value0.00*). Conclusion: Hamstring technique showed good outcomes which can be used to reconstruct ACL for better quality lifestyle

Key words: $\quad$ ACL, Hamstring, Osteoarthritis, Tegnors Scale.

Article Citation: Khan ZA, Altaf B, Javed S, Altaf S, Jawed S. Functional outcomes of ACL reconstruction using hamstring auto graft. Professional Med J 2019; 26(11):1965-1970. DOI: 10.29309/TPMJ/2019.26.11.3660

\section{INTRODUCTION}

The Anterior Cruciate Ligament (ACL) is one the important ligament of the knee which is thought to play a major role to stabilize the knee. The main function of the ACL is to govern anterior translation of tibia with subsequent internal rotation of the knee. ${ }^{1}$ This is possible not only because of ACL but also because of presence of other articular structures which are also believed to be associated with ACL for the purpose of sustaining knee stability. It is known very well regarding the $A C L$ formation that it is comprised of two different sets of bundles one of which is the antro-medial and the other one is the postero-lateral. Though both of these owe different functions but important thing regarding these sets of bundles is that they work in unity. Now a days ACL injury is seen very commonly, especially among athletes. Irrespective of the positive effects and outcomes of sports on health, the risk for injury also remains a serious issue for young athletes. Growing evidence are available showing that $A C L$ injuries are responsible for causing osteoarthritis at a later age. Hence, it can be taken as one of the major risk factor for osteoarthritis. Shea et al. reported that knee injuries account for 13.2 percent of all sports injuries, of which the vast majority are tears of $A C L$. Tears of the anterior cruciate ligament (ACL) in young athletes have increased over the past 20 years. In the literature, ACL gaps have been reported for 250,000-300,000 people per year. 
However, the injury rate of ACL in comparable sports in basketball and football has remained frighteningly high with the change in the ratio between men and women over the past 10 years. The incidence among women is 3.5 times higher in basketball and 2.8 times higher in football. A typical injury mechanism is a quick but awkward stopping and waiting for lateral movements. It has been reported that gender differences in neuromuscular activation patterns contribute to ACL damage. When it comes to compare with men, it is seen that women are less effective in strengthening the knee. Maximum contraction of the knee musculature significantly reduces anterior translation of the tibia in men and women compared with relaxed and contracted states. ${ }^{2}$

Women experience $\mathrm{ACL}$ tears up to nine times more often than men. ${ }^{3}$ This high incidence in females may related due to the increasing trend of girls towards sports. The ACL can stretch or tear in case of any sharp turn during running or because of any sudden quick movement. It is quiet painful condition, and subject is unable to put all his weight on the effected limb thence he/she may suffer from difficult walking. For treatment purpose, anterior cruciate ligament repair and reconstruction is done with the help of surgery. It is one of the most common surgeries in developed countries. In US, the annual incidence of $A C L$ reconstruction is reported to be 36.9 per 100,000 subjects. Now a days the techniques that are being widely used involves patellar tendon autograph technique and hamstring auto-grafts technique. Though, both these reconstruction techniques have very little differences in procedure but over the last decade, auto-grafts with help of arthroscopic hamstring is becoming the popular procedure for $A C L$ repair and reconstruction. Data of developed countries is available showing auto-grafts using hamstring have been increased from $68 \%$ in 2005 to almost $85 \%$ in $2011 .{ }^{4}$ Patellar tendon auto-grafts are seen to be associated with knee pain which often involves the anterior part of knee while hamstring auto-grafts tend to evolve towards greater residual laxity. Thus, the choice for choosing the technique solely depends upon surgeon's preference owing to its own experience. Couple of researches are available showing that further surgeries were needed in the subjects whose ACL were repaired using the hamstring autograph technique as compared to patellar tendon grafts ${ }^{5,6}$, however recent studies $^{7,8}$ did not find evidence supporting this. The rationale of this study is to determine the functional outcome of arthroscopic ACL reconstruction with hamstring technique by evaluating Tegner activity score. Finding a reliable technique will help us in making a standard protocol for reconstruction of $A C L$. We will see the functional outcome of arthroscopic ACL reconstruction using hamstring technique. If our results are satisfactory we can use this technique as gold standard in repairing all cases of ACL. In this way this study will help us to reduce the morbidity associated with this debilitating condition and will improve quality of life in patients of ACL rupture.

\section{OBJECTIVE}

To determine the functional outcome of arthroscopic ACL reconstruction with hamstring technique by evaluating Tegner activity score.

\section{Data Collection Procedure}

A cross sectional study was carried out after approval from hospital ethical committee. It was comprised of 97 patients. Sample size was calculated with Power 80 , prevalence $20 \%$ and $95 \%$ of $\mathrm{Cl}$ with open EPI-sample size calculate for cross sectional study. Written informed consent from each patient who had an isolated ACL rupture of 17 to 68 years (inclusion criteria) Patients with comorbidity, or any previous surgery to the affected knee, multi-ligamentous injuries, previous surgery to same knee or having osteoarthritis were excluded. Their demographic features i.e. name, age, gender, and address is recorded. History was taken about the pain in knee, mechanism and duration of injury. Diagnosis was made primarily on the basis of examination following positive anterior draw test and Pre-operative Tegner activity rating scale is calculated for all patients. Preoperative physical findings and radiographs record was maintained. One surgeon with the help of the same operating team and instruments performed all the operations using autologous STG tendon graft. Postoperatively all patients were subjected 
to a standardized rehab protocol for 6months after which final readings were taken. The affected knee joint was thoroughly examined under anaesthesia, knee instability findings were confirmed and documentation was done. Anterolateral and anteromedial ports used for knee arthroscopy. Femoral and tibial footprints and tunnels were prepared. STG tendons were harvested using tendon stripper, maximum tension was given using Naseer Awais (NA) fixator for five minutes, then tendon was folded four times and fixed in place using bioabsorbable screw and vicryl \#1 suture. A standardized postoperative rehabilitation protocol was given to all patients and it was supervised by two personnel and compliance was documented. For controlled mobilization, knee braces were used for the first three weeks postoperatively. Assisted partial weight bearing was allowed in the first three postoperative weeks. At the last follow-up, ROM, stability and knee proprioception was measured against the healthy side. Statistical analysis was done using SPSS 17. Descriptive statistics, Student $t$ test (paired or unpaired), chi-square analysis, Fisher exact tests were appropriately applied. Significance was established at $p<0.05$. All patients will underwent arthroscopic $A C L$ reconstruction using hamstring autograph technique.

\section{Surgical procedure}

The surgery was performed by single surgeon. Tourniquet was used in all cases. Standard anteromedial (AM) and anterolateral (AL) portals were used to perform the arthroscopic inspection of the knee joint to confirm the diagnosis and look for associated meniscal or chondral lesions. Then torn ACL is carefully removed with special attention to the anatomic footprints of the 2 ACL bundles. A transtibial technique was used to create femoral tunnel for ACL double-bundle reconstruction. The guide wire was passed through the tibial tunnel and tip of the guide wire was placed on the femoral footprint of the AM bundle. AM femoral tunnel was drilled to a depth of $35 \mathrm{~mm}$ to $40 \mathrm{~mm}$. The far cortex of the AM femoral tunnel was breached with a $4.5-\mathrm{mm}$ drill bit. Once the femoral socket has been fashioned with concentric reaming, a marking hook, firmly mounted on the femoral guide, was introduced. The hook (which matches the socket diameter) is pushed in the socket. A guide pin was drilled through the distal femur, from lateral to medial. The guide pin was them replaced by a guide wire, which is brought out through the tibial tunnel. The graft was loaded into the wire loop. Rigidfix was slided over the wire, through the axilla of the graft, and impacted into the lateral femoral cortex. First, the tendon grafts were trimmed and the diameters of the double-stranded grafts were adjusted. The AM tendon graft was trimmed such that the diameter of the double-stranded graft is 8 $\mathrm{mm}$ to $9 \mathrm{~mm}$; the PL graft was trimmed such that the diameter of the double stranded graft is $7 \mathrm{~mm}$ to $8 \mathrm{~mm}$. A Beath pin with a long looped suture attached to the eyelet was passed through the accessory anteromedial portal and out through the PL femoral tunnel. The looped suture was visualized within the joint and retrieved with an arthroscopic suture grasper through the PL tibial tunnel. On the tibial side, we used a bio absorbable interference screw fixation. The PL bundle graft was tensioned and fixed between $0^{\circ}$ to $10^{\circ}$ of flexion. The AM bundle graft was tensioned and fixed between $60^{\circ}$ and $90^{\circ}$ of flexion.

Post-operative care was done in orthopedic ward and patient discharged next day according to hospital standards. Application of icepacks to relieve discomfort and antibiotics to avoid infection, along with analgesics according to need had been advised. Weight bearing allowed post operatively with knee immobilizer as pain tolerated and quadriceps strengthening exercises had been advised. Patients were called after 2 weeks of surgery to remove stitches and regular physiotherapy of knee advised to gradually achieve full range of motion. Patients were called after 12 weeks of surgery and post-operative Tegner's activity rating scale was calculated to evaluate the improvement. Patient was labeled as improved whose post-operative Tegner's rating scale was more than six. All data was collected through a well-defined proforma attached.

\section{Data Analysis Procedure}

Data will be entered on software SPSS version 23.0. Quantitative data like age, Tegner score and 
post-operative Tegner's score was presented by mean and standard deviation while qualitative data like gender and improvement after surgery will be presented by frequency and percentages. Data was stratified for the variables i.e. age, gender, and pre-operative Tegner activity rating scale to address the effect modifiers. Poststratification Chi-square test is applied to check the significance with P-value less than 0.05 as significant. Regression was applied between the age and Tegnor's sacle.

\section{RESULTS}

This study comprised of 97 subjects (81 male, 16 females) $83.5 .5 \%$ and $16.5 \%$ respectively from private setup based hospital with mean age

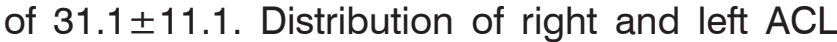
damage in our studied population is shown in the figure-1. Showing that right sided presentation is more common than the left side. Table-I. Shows that $79(97.5 \%)$ of the males have damaged their right sided ACL while only 2 (2.5\%) were presented with left sided ACL damage. In contrast to this $16(100 \%)$ presented with right side and left sided ACL damage respectively. Figure 2 shows the anterior drawer test in post-operative subject.

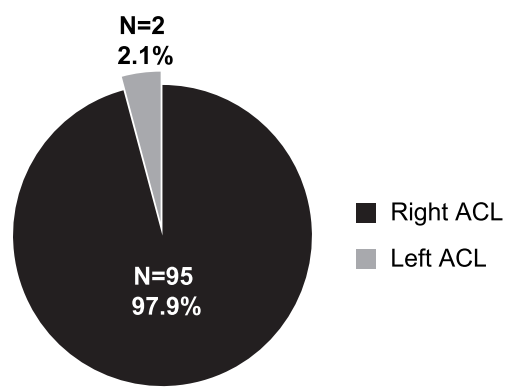

Figure-1. Percentage of distribution of ACL damage effecting right or left side

Percentages of subject showing Anterior drawer test

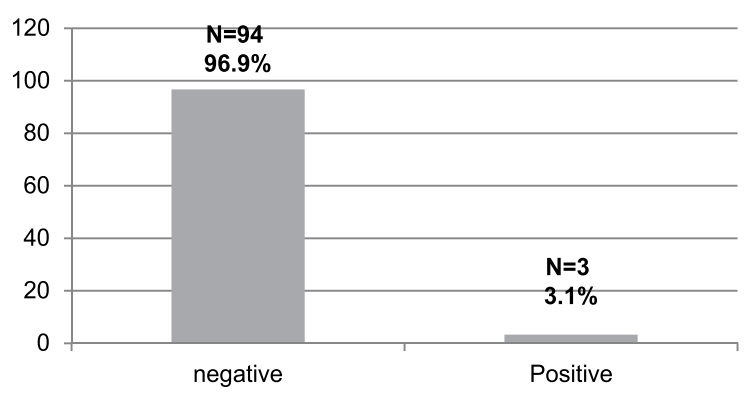

Figure-2. Percentage of subjects showing postoperative anterior drawer test activity

\begin{tabular}{|c|c|c|c|}
\hline $\begin{array}{l}\text { Damaged Aide } \\
\qquad \mathrm{N}=97\end{array}$ & Male & Female & P-Value \\
\hline Right ACL & 79 (97.5\%) & $16(100 \%)$ & \multirow{2}{*}{0.328} \\
\hline Left ACL & $2(2.5 \%)$ & 0 & \\
\hline \multicolumn{4}{|c|}{$\begin{array}{l}\text { Table-I. Percentages of gender showing damage of } \\
\text { right and left sided involvement }\end{array}$} \\
\hline \multicolumn{2}{|c|}{ Tegner's Activity Score } & \multicolumn{2}{|c|}{ Frequency $\mathrm{N}=97$} \\
\hline \multicolumn{2}{|c|}{$\leq 6$} & \multicolumn{2}{|c|}{$94(96.9 \%)$} \\
\hline \multicolumn{2}{|l|}{$\geq 5$} & \multicolumn{2}{|c|}{$3(3.1 \%)$} \\
\hline \multicolumn{2}{|l|}{ Total } & \multicolumn{2}{|c|}{97} \\
\hline \multicolumn{4}{|c|}{ Table-II. Tegner's Acore } \\
\hline & \multicolumn{2}{|l|}{ B } & -Value \\
\hline & \multicolumn{2}{|l|}{$-3.5 .2$} & $.000^{\star}$ \\
\hline \multicolumn{4}{|c|}{$\begin{array}{l}\text { Table-III. Regression analysis for age and post- } \\
\text { operative. Tegnrs activity score } P \text { value } \leq 0.05 \\
\text { considered to be significant }\end{array}$} \\
\hline
\end{tabular}

To check the effect of age on outcomes of Tegners scorre, chi-square test was applied which was found to be significant ( $P$ value $0.000 *$ ). However results of chi-square for gender affecting Tegner's activity were found non-significant with $P$ value 0.57. Table-II. Shows that 94 (96.9\%) subjects improved their Tegne'rs activity score more than 6 post-operative while only $3(3.1 \%)$ showed Tegner's score less than 6 post operatively which was though improved but utilizing more rehabilitation techniques and more duration. However, it is seen that results were found to be significant with $P$ value 0.000 * when it comes to check that the subject who were having postoperative anterior drawer test not very much improved also showed poor improvement in Tegners activity score. Table-III showed regression analysis indicating that increase in one year of the age will decrease 3.5 times reduction in Tegnors score * $\left(p\right.$ value $\left.0.000^{*}\right)$.

\section{DISCUSSION}

Haearthosis is upto $70 \%{ }^{1}$. Studies showed that if ACL tears are managed conservatively there is increased incidence of meniscal tears and early arthritic changes in the knee joint. ${ }^{2}$ The reason to repair a torn $A C L$ is to provide knee stability, painless range of motion and to prevent osteoarthritis. The gold standard for ACL autograph reconstruction is bone patella-tendon 
bone (BTB). ${ }^{3}$ However, there are controversies regarding the optimal timing of surgery. Some studies report increased knee stiffness if the surgery is done early after $A C L$ reconstruction, but some studies have reported good results after early repair of the torn ligament. ${ }^{4}$ The most common side effect of using BTB graft is anterior knee pain. ${ }^{5}$ The BTB graft heals when bone plug is united, yielding higher strength of healing. ${ }^{6}$ Other graft options The knee joint consists of two cruciate ligaments. Out of the two ligaments, the anterior cruciate ligament (ACL) is much weaker compared with the posterior cruciate ligament (PCL). ACL tears are most often overlooked. The incidence of ACL tear in patients suffering from haearthosis is up to $70 \% .^{1}$ Studies showed that if $\mathrm{ACL}$ tears are managed conservatively there is increased incidence of meniscal tears and early arthritic changes in the knee joint. ${ }^{2}$ The reason to repair a torn $A C L$ is to provide knee stability, painless range of motion and to prevent osteoarthritis. The gold standard for ACL autograph reconstruction is bone patella tendon bone (BTB). ${ }^{3}$

However, there are controversies regarding the optimal timing of surgery. Some studies report increased knee stiffness if the surgery is done early after ACL reconstruction, but some studies have reported good results after early repair of the torn ligament. ${ }^{4}$ The most common side effect of using BTB graft is anterior knee pain. ${ }^{5}$ The BTB graft heals when bone plug is united, yielding higher strength of healing. ${ }^{6}$ Other graft options. The knee joint consists of two cruciate ligaments. Out of the two ligaments, the anterior cruciate ligament $(A C L)$ is much weaker compared with the posterior cruciate ligament (PCL). ACL tears are most often overlooked. The incidence of ACL tear in patients suffering from haearthosis is upto $70 \% .1$ Studies showed that if $\mathrm{ACL}$ tears are managed conservatively there is increased incidence of meniscal tears and early arthritic changes in the knee joint. The reason to repair a torn ACL is to provide knee stability, painless range of motion and to prevent osteoarthritis. The gold standard for $A C L$ autograft reconstruction is bone patella-tendon bone (BTB). ${ }^{3}$ However, there are controversies regarding the optimal timing of surgery. Some studies report increased knee stiffness if the surgery is done early after ACL reconstruction, but some studies have reported good results after early repair of the torn ligament. ${ }^{4}$ The most common side effect of using BTB graft is anterior knee pain. ${ }^{5}$ The BTB graft heals when bone plug is united, yielding higher strength of healing. ${ }^{6}$ Other graft options. Knee joint is comprised of two important ligaments one is the anterior cruciate ligament while the other one is posterior cruciate ligament. When there is damage to anterior cruciate ligament then symptoms don't appear in some patients until they experience any activity like playing or landing at acute angle. Our study comprised of 97 subjects who were involved in sports activity. Out of this 93 subject faced ACL injury during a play while 4 subjects were those who came with RTA.

It is documented by some researchers that if ACL reconstruction is not done timely then it can ultimately results in unacceptable outcomes like menisci problems or osteoarthritis etc. ${ }^{9}$ Asik et al. Observed hamstring tendons ACL reconstruction mid to long-term outcomes in 271 patients with anterior cruciate ligament ruptures. He stated $24 \%$ subjects with improved Tegner activity level scale which was more than 6 postoperatively. ${ }^{9}$ However, Shervegar $\mathrm{S}$ and his collegues stated that they found more than $90 \%$ satisfaction rate comparable to studies of Asik etal for ACL reconstruction using hamstring. In agreement to this study, our studied also the similar results showing good outcomes for Tegner's activity with good range of movement after reconstruction of ACL with hamstring. We did not look for followup. It is advisable for follow-up at least for 5 years especially for those subject in age of 40 s which show good out comes without any post-surgery tears which may come across with upcoming 5 years of surgery..$^{10}$ Incontrast to this, studies are available that show that 14 years to 29 years of age group is best suited for ACL reconstruction. Who are undergoing for post traumatic $A C L$ reconstruction. Data is available showing that subjects of these age groups show good outcomes. ${ }^{11}$ So it is advisable to check postsurgery $\mathrm{ACL}$ reconstruction long term outcomes 
for specific age group.

\section{CONCLUSION}

Good Tegners score was found in ACL reconstruction done by Hamstring auto graph technique indicating good outcomes. Hence this technique can be used for better quality lifestyle. Copyright@ 05 July, 2019.

\section{REFERENCES}

1. Ferretti $A$, Monaco $E$, Vadalà $A$. Rotatory instability of the knee after ACL tear and reconstruction. Journal of Orthopaedics and Traumatology. 2013; 15(2):75-79.

2. Collins SL, Layde P, Guse CE, Schlotthauer AE, Van Valin $\mathrm{SE}$. The Incidence and Etiology of Anterior Cruciate Ligament Injuries in Patients under the Age of 18 in the State of Wisconsin. Pediat Therapeut 4: 196. doi: 10.4172/2161-0665.1000196 Page 2 of 4 Pediat Therapeut ISSN: 2161-0665 Pediatrics, an open access journal Volume 4 • Issue 2• 1000196. Figure. 2014;2:0-17.

3. The female ACL: Why is it more prone to Injusry? J Orthop. 2016; 13(2): A1-A4

4. Rahr-Wagner L, Thillemann T, Pedersen A, Lind M. Comparison of hamstring tendon and patellar tendon grafts in anterior cruciate ligament reconstruction in a nationwide population-based cohort study. The American Journal of Sports Medicine. 2013; 42(2):278284.

5. Gifstad T, Foss O, Engebretsen L, Lind M, Forssblad $M$, Albrektsen $G$ et al. Lower risk of revision with patellar tendon autografts compared with Hamstring Autografts. The American Journal of Sports Medicine. 2014; 42(10):2319-2328.
6. Persson A, Fjeldsgaard K, Gjertsen J, Kjellsen A, Engebretsen L, Hole $R$ et al. Increased risk of revision with hamstring tendon grafts compared with patellar tendon grafts after anterior cruciate ligament reconstruction. The American Journal of Sports Medicine. 2013; 42(2):285-291.

7. Gabler C, Jacobs C, Howard J, Mattacola C, Johnson D. Comparison of graft failure rate between autografts placed via an anatomic anterior cruciate ligament reconstruction technique. The American Journal of Sports Medicine. 2015; 44(4):1069-1079.

8. Xie X, Liu X, Chen Z, Yu Y, Peng S, Li Q. A meta-analysis of bone-patellar tendon-bone autograft versus fourstrand hamstring tendon autograft for anterior cruciate ligament reconstruction. The Knee. 2015; 22(2):100110.

9. Asik M, Sen C, Tuncay I, Erdil M, Avci C, The mid- to long-term results of the anterior cruciate ligament reconstruction with hamstring tendons using Transfix technique. Knee Surg Sports Traumatol Arthrosc. 2007; 15(8):965-72.

10. Brown CA, McAdams TR, Harris AH, Maffulli N, Safran MR. ACL reconstruction in patients aged 40 years and older: a systematic review and introduction of a new methodology score for ACL studies. American Journal of Sports Medicine 2013; 41(9): 2181-2190. [PubMed]

11. Magnusson J, Podesta L, etal. Anterior cruciate ligament reconstruction rehabilitation for the postsurgical orthopedic patient. 2013; 3:404-426. https://doi. org/10.1016/B978-0-323-07747-7.00022-8

12. Sue D. Barber-Westin, Frank R. Noyes, Rating of athletic and daily functional activities, Noyes' Knee disorders: Surgery, rehabilitation, clinical outcomes. 2017; 2, ISBN 978-0-323-32903-3

13. Shervegar S, Nagaraj P, Grover A, Dj NG, Ravoof A. Functional outcome following arthroscopic acl reconstruction with rigid fix: A retrospective observational study. Arch Bone $\mathrm{Jt}$ Surg. 2015; 3(4):264-8.

\section{AUTHORSHIP AND CONTRIBUTION DECLARATION}

\begin{tabular}{|c|c|c|c|}
\hline Sr. \# & Author(s) Full Name & Contribution to the paper & Author(s) Signature \\
\hline 1 & Zeeshan Ali Khan & $\begin{array}{l}\text { Contributed to acquisition, he is the guarantor } \\
\text { of this work and as such had full access to all } \\
\text { the data in the study and takes responsibility for } \\
\text { the integrity of the data and the accuracy of the } \\
\text { data analysis. }\end{array}$ & \\
\hline 2 & Benash Altaf & $\begin{array}{l}\text { Contributed to study design, wrote the } \\
\text { manuscript and reviewed references. She also } \\
\text { contributed to data analysis, interpretation, } \\
\text { editing and formatting the manuscript. Read } \\
\text { and approval the manuscript. }\end{array}$ & \\
\hline 3 & Samia Javed & Read and approved it. & \\
\hline 4 & Sadaf Altaf & $\begin{array}{l}\text { Contributed in editing and formatting the } \\
\text { manuscript. Reviewed and approved the }\end{array}$ & \\
\hline 5 & Shireen Jawed & $\begin{array}{l}\text { manuscript. } \\
\text { Controbuted in data analysis and interpretation. }\end{array}$ & \\
\hline
\end{tabular}

